choroiditis and less frequently papillitis or optic neuritis. Our patient presented with simultaneous reactivation of two areas of choroidoretinitis, characteristic of toxoplasmosis, which could be related to the loss of visual acuity and the visual field defect. However, the painful eye movements and the afferent pupillary defect were more suggestive of optic nerve disease. ${ }^{78}$ This was confirmed by an MRI examination which revealed an active inflammatory lesion in mid-portion of the right optic nerve, separate from the retinal lesions. Such optic nerve lesions occur in demyelinating disease, 59 in granulomatous disease and in Leber's hereditary optic neuropathy, ${ }^{10}$ which is usually situated more posteriorly.

However, the optic nerve lesion is more likely to have resulted from an active toxoplasmic lesion, particularly as there were no other features of demyelination.Toxoplasma gondii has been isolated from the optic nerves at post morten in an infant with congenital toxoplasmosis, ${ }^{1}$ a patient receiving long term steroids, ${ }^{4}$ and in two patients with AIDS. ${ }^{23}$ Rarely, the cysts are associated with inflammation and demyelination of the optic nerve."

The present report is unique in describing an immunocompetent adult who, in addition to active retinal toxoplasmosis, had a simultaneously active retrobulbar optic nerve lesion on MRI. This is the first in vivo report of such an association, raising the possibility that optic nerve lesions may be more common in cases of typical toxoplasmic retinochoroiditis than previously considered, and that MRI may be valuable if visual function does not correlate with the clinical presentation or with the topography of the retinal lesions.

We thank Professor W I McDonald for permission to report the case.

Dr F-X Borruat was supported by private funds from the Hopital Ophtalmique Jules Gonin, University Eye Clinic of Lausanne, Switzerland, and by grants from the Societe Academique Vaudoise, Fondation Florian Verrey, Fondation SICPA, Fondation due 450eme anniversaire de l'Universite de Lausanne.

1 Manschot WA, Daamen CBF. Connatal ocular toxoplasmosis. Arch Ophthalmol 1965; 74: 48-54.

2 Holland GN, Engstrom RE, Glasgow BJ, Berger BB, Daniels SA, Sidikaro Y, et al. Ocular toxoplasmosis in patients with the acquired immunodeficiency syndrome. Am 7 Ophthal mol 1988; 106: 653-67.

3 Grossniklaus HE, Specht CS, Allaire G, Leavitt JA. Toxoplasma gondii retinochoroiditis and optic neuritis in acquired immune deficiency syndrome. Ophthalmology 1990; 97: 1342-6

4 Nicholson DH, Wolchok EB. Ocular toxoplasmosis in an adult receiving long-term corticosteroid therapy. Arch Ophthalmol 1976; 94: 248-54.

5 Miller DH, Johnson G, McDonald WI, MacManus D, du Boulay EPGH, Kendall BE, et al. Detection of optic nerve lesions in optic neuritis with magnetic resonance imaging. Lancet 1986; i: 1490-1.

6 Hawkins CP, Munro PMG, Mackenzie F, Kesselring J, Tofts PS, du Boulay EPGH, et al. Duration and selectivity of blood-brain barrier breakdown in chronic relapsing blood-brain barrier breakdown in chronic relapsing experimental allergic encephalomyelitis studied by gadolinium

7 Tabara KF. Toxoplasmosis. In: Tasman W, Jaeger EA, eds. Duane's clinical ophthalmology. Vol 4. External diseases of the uvea. Philadelphia: Lippincott, 1991: Ch 46, 123.

8 Folk JC, Lobes LA. Presumed toxoplasmic papillitis. Ophthalmology 1984; 91: 64-7.

9 Miller DH, Newton MR, van der Poel JC, du Boulay EPGH, Halliday AM, Kendall BE, et al. Magnetic resonance imaging of the optic nerve in optic neuritis. Neurology 1988; 38: 175-9.

10 Kermode AG, Moseley IF, Kendall BE, Miller DH, MacManus D, McDonald WI. Magnetic resonance imaging in Leber's optic neuropathy. F Neurol Neurosurg Psychiatry 1989; 52: 671-4.

11 De Girolami U, Hénin D, Girard B, Katlama C, Le Hoang P, Hauw JJ. Etude pathologique de l'oeil et du système nerveux central dans 25 cas de SIDA. Rev Neurol (Paris) 1989; 145: 819-28.

\title{
Focal electroretinogram and visual field defect in multiple evanescent white dot syndrome
}

\author{
Masayuki Horiguchi, Yozo Miyake, Makoto Nakamura, Yukari Fujii
}

\author{
Department of \\ Ophthalmology, Nagoya \\ University School of \\ Medicine, Japan \\ M Horiguchi \\ Y Miyake \\ M Nakamura \\ Y Fujii \\ Correspondence to: \\ Masayuki Horiguchi, MD, \\ Department of \\ Ophthalmology, Nagoya \\ University School of \\ Medicine, 65 Tsuruma-cho, \\ Showa-ku, Nagoya 466, \\ Japan. \\ Accepted for publication \\ 10 February 1993
}

Multiple evanescent white dot syndrome (MEWDS) is characterised by decreased visual acuity, white dots at the level of retinal pigment epithelium (RPE), scotoma, including enlargement of the blind spot, and visual return without treatment. ${ }^{12}$ Although electrophysiological data, such as reduced a-wave in electroretinogram (ERG) and reduced early receptor potential, ${ }^{2}$ indicate that the disease primarily affects the RPE and the outer segments of photoreceptors, optic nerve involvement has been reported to be a possible cause of the scotoma. ${ }^{3}$ On the other hand, several reports ${ }^{467}$ suggest that a disease known as 'acute idiopathic blind spot enlargement (AIBSE)', 8 in which the enlargement is caused by retinal dysfunction, and MEWDS are the same clinical entities. We recorded focal electroretinographs in two patients with MEWDS to study the nature of the scotoma.

\section{Case reports}

CASE 1

A 32-year-old woman noticed a central scotoma in the left eye, and was examined 6 days after the onset. Corrected visual acuity was $1 \cdot 2$ right eye and 0.2 left eye. Fundus examination of the left eye revealed multiple white lesions deep in the retina and fluorescein angiography demonstrated 
Actual (dB)

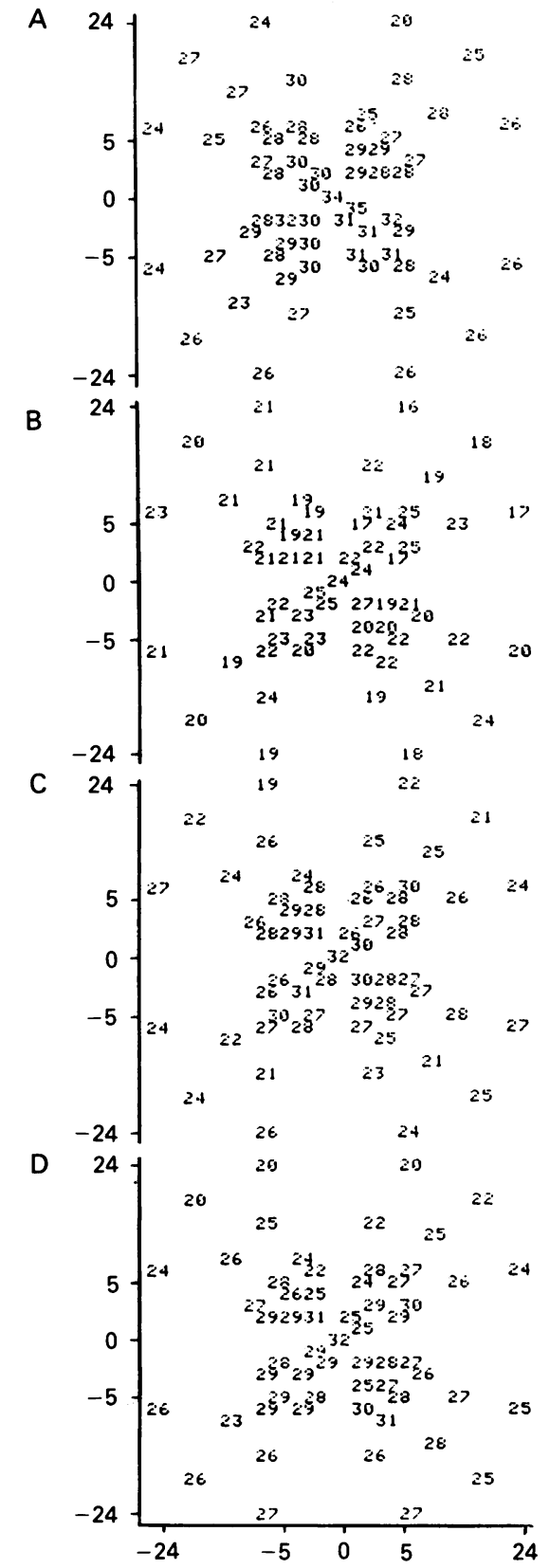

Figure 1 The Octopus $M$ program in case 1 . (A) $A$ normal left eye. (B) Right eye at initial visit. The threshold was remarkably elevated. (C) Left eye 1 month after initial visit. The threshold in the central portion was slightly elevated as compared with normal left eye. (D) Left eye 12 months after initial visit. The central threshold was still slightly elevated.
TC: $0.03 \mathrm{~s}$

TC: $0.003 \mathrm{~s}$

A
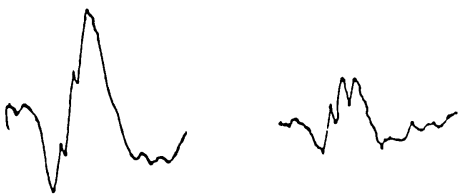

B

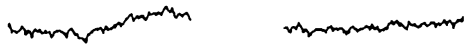

C

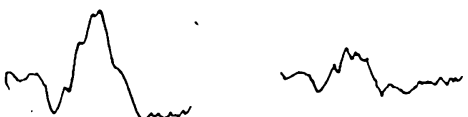

D

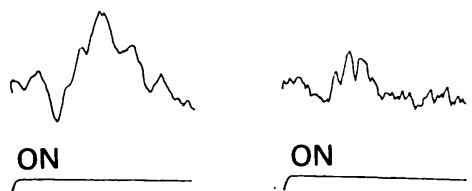

$1 \mu \mathrm{V}$

Figure 3 Focal macular ERG (spot size: $15^{\circ}$ ) in case 1. (A) $A$ normal left eye. $(B) R$ Right eye at initial visit. The focal $E R G$ was deteriorated. (C) Left eye 1 month after initial visit. The amplitudes of $a$ - and $b$-waves and ocillatory potentials were slightly reduced. (D) Right eye 12 months after initial visit. The amplitudes of all components were still slightly reduced.

patchy hyperfluorescence in the posterior pole. Psychophysical threshold was elevated in the central field of the left eye (Octopus Program M1) (Fig 1A (left eye), 1B (right eye)). While full field ERG, recorded using the standard technique reported previously, ${ }^{9}$ showed a slight reduction in amplitudes (Fig 2A (left eye), 2B (right eye)), focal macular ERG (spot size $15^{\circ}$ ) was nonrecordable in the left eye (Fig 3A (left eye), Fig 3B (right eye)). The details of our method for recording focal ERG have been described elsewhere. ${ }^{1011} 12$

One month later, visual acuity of the left eye recovered to $1 \cdot 2$, and the psychophysical threshold in the macula was only slightly elevated (Fig 1C). Fundus abnormalites had disappeared, and full field ERG was normal except for a very slight reduction of the scotopic component (Fig 2C). However, the amplitudes of a- and b-waves and oscillatory potentials (OPs) in focal macular ERG remained reduced (Fig $3 \mathrm{C}$ ).

Twelve months later the central threshold was still slightly elevated (Fig 1D), and the amplitudes of all components in focal macular ERG remained abnormal (Fig 3D).

\section{CASE 2}

A 24-year-old man reported a 5 day history of visual disturbance. Corrected visual acuity was 1.5 right eye and 0.4 left eye. Fundus examination of the left eye revealed multiple white dots deep in the retina and fluorescein angiography showed early hyperfluorescence in the macula and dye leakage from the disc. Goldmann perimetry revealed an enlarged blind spot (Fig 4A (right eye), 4B (left eye)) in the left eye. Focal ERG recorded beside the optic disc (spot size $10^{\circ}$ ) had deteriorated (Fig 5A (right eye), 5B (left eye)), while the amplitudes of focal macular ERG (spot size $10^{\circ}$ ) were slightly reduced (Fig 6A (right eye), 6B (left eye)).

Figure 2 The full field ERG in case 1. (A) A normal left eye. (B) Right eye at initial visit. All components showed a slight reduction in the amplitude. (C) Right eye I month after initial visit. The ERG was normal except for a slightly reduced scotopic b-wave. 


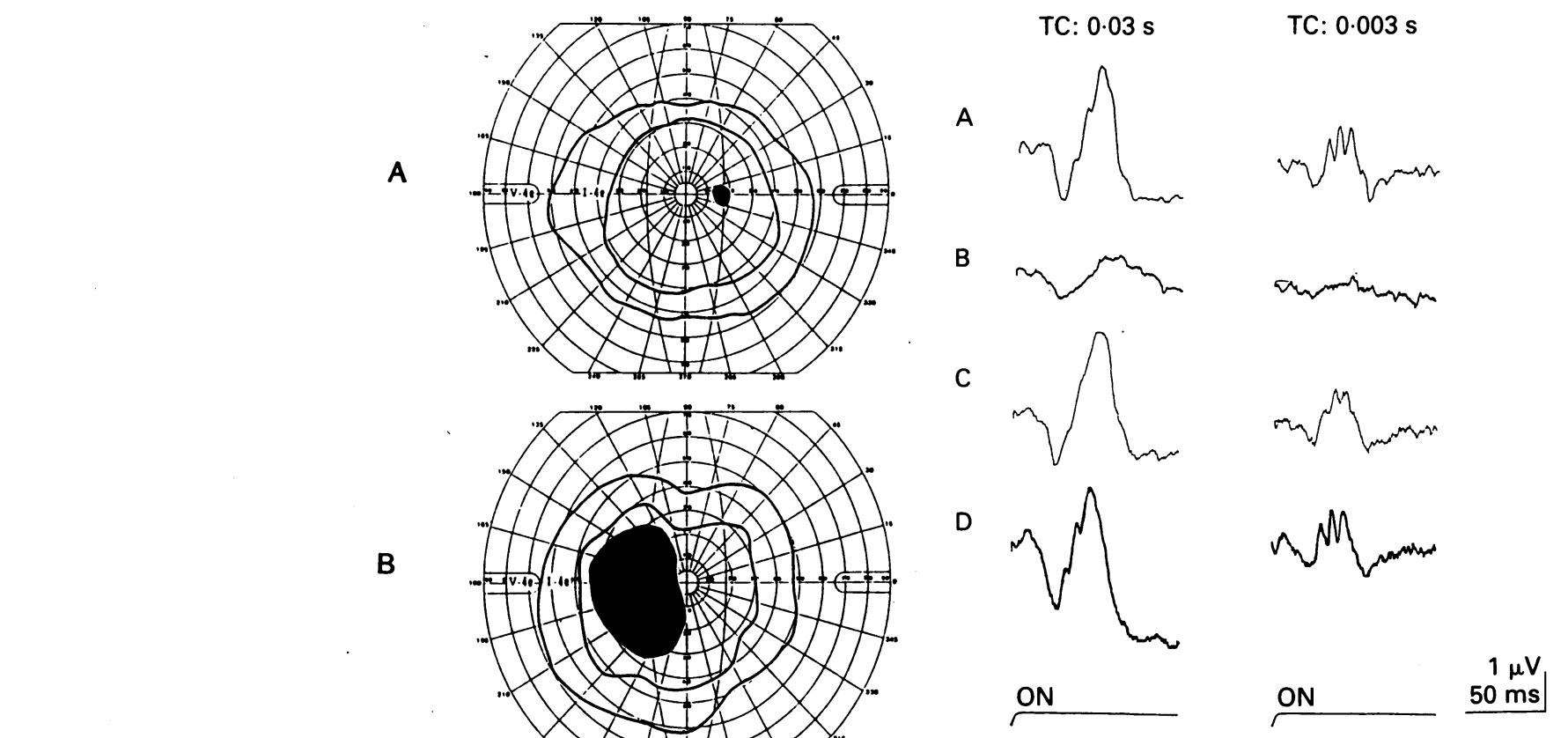

Figure 4 Goldmann perimetry in case 2 . (A) $A$ normal right eye. (B) Left eye at initial visit. The blind spot was enlarged. (C) Left eye 2 months after initial visit. The enlargement was minimised. (D) Left eye 14 months after initial visit. The size of the blind spot was almost identical to that in the right eye.
Figure 5 Focal ERG recorded from the area between the optic disc and fovea (spot size: $10^{\circ}$ ) in case 2. (A) Normal right eye. (B) Left eye at initial visit. The focal ERG was almost flat. (C) Left eye 2 months after initial visit. The amplitudes of $a$ - and $b$-wave and oscillatory potentials were reduced compared with those in the normal right eye. (D) Left eye 14 months after initial visit. The amplitude of all components were still reduced.
C
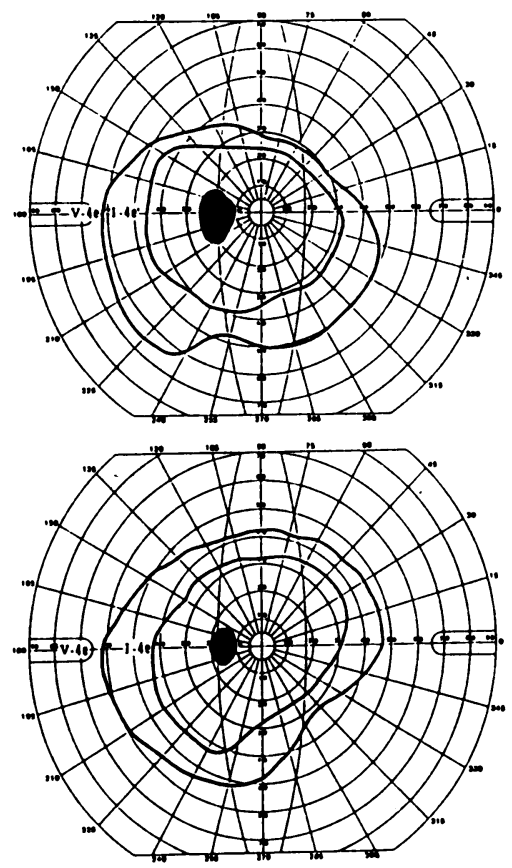

Figure 6 Focal ERG recorded from the macula (spot size: $10^{\circ}$ in case 2. (A) Normal right eye. (B) Left eye at initial visit. The amplitudes of $a$ - and $b$-waves and oscillatory potentials were reduced. (C) Left eye 2 months after initial visit. The amplitudes of $a$-and $b$-waves were normal, but that of oscillatory potentials was still reduced. (D) Left eye 14 months after initial visit. The amplitudes of all components were normal.

Two months later, the corrected visual acuity in the left eye returned to $1 \cdot 0$, and the enlargement of the blind spot had minimised (Fig 4C). Fundus examination and fluorescein angiography revealed no abnormality. The amplitudes of all components in focal ERG beside the optic disc were slightly reduced (Fig $5 \mathrm{C}$ ), whereas in the macula the amplitudes of a- and b-waves were normal but those of OPs were reduced (Fig $6 \mathrm{C})$.

Fourteen months later, the enlargement of the blind spot had almost disappeared (Fig 4D). All components in focal ERG remained abnormal beside the disc (Fig 5D), while they returned completely to normal in the macula (Fig 6D).

\section{Comment}

Although Dodwell et $\mathrm{al}^{3}$ believed that optic nerve dysfunction produces scotoma in MEWDS, several authors ${ }^{467}$ suggested that AIBSE where multifocal study proved the retinal nature of scotoma $^{8}$ is a subset of MEWDS. Sigh et $a l^{13}$ considered that NEWDS is a subset of AIBSE. The nature of scotoma in MEWDS needed to be studied before any conclusion can be drawn.

In case 1 with central scotoma, the amplitude was more reduced in macular ERG than in full field ERG, and its recovery was much slower in the former. In case 2 with blind spot enlargement, the amplitude of focal ERG was more reduced beside the optic disc than in the macula, and the recovery of the amplitude slower beside the optic disc. These data indicate that the scotoma was caused by retinal dysfunction in our patients. Furthermore, the results of case 2 support the hypothesis of clinical entities being similar between MEWDS and AIBSE. Finally, our ERG technique, totally different from the
D

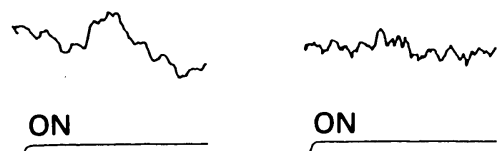


multifocal ERG ${ }^{8}$ recorded a- and b-waves and OPs, and demonstrated delayed recovery of OPs in case 2, which may imply inner retinal layer involvement in MEWDS. Similar findings have been reported in central serous chorioretinopathy. ${ }^{14}$

1 Jampol LM, Sieving PA, Pugh D, Fishman GA, Gilbert H. Multiple evanescent white dot syndrome: I. Clinical Multiple evanescent white dot syndrom

2 Sieving PA, Fishman GA, Jampol LM, Pugh D. Multiple evanescent white dot syndrome: II. Electrophysiology of the evanescent white dot syndrome: II. Electrophysiology of the

3 Dodwell DG, Jampol LM, Rosenberg M, Berman A, Zaret CR. Optic nerve involvement associated with the multiple evanescent white-dot syndrome. Ophthalmology 1990; 97: 862-8.

4 Hamed LM, Glaser JS, Schatz NJ, Gass DM. Acute idiopathic blind spot enlargement without optic disc edema. Arch Ophthalmol 1988; 106: 1030-1.

5 Hamed LM, Glaser JS, Gass DM, Schatz NJ. Protracted enlargement of the blind spot in multiple evanescent white dot syndrome. Arch Ophthalmol 1989; 107: 194-8.
6 Aarberg TM. Multiple evanescent white dot syndrome. Arch Ophthalmol 1988; 106: 1162-3.

7 Kimmel AS, Folk JC, Thompson HS, Strand LS. The multiple evanescent white dot syndrome with acute blind spot enlargement. Am $\mathcal{F}$ Ophthalmol 1989; 107: 425-6.

8 Fletcher WA, Imes RK, Goodman D, Hoyt WF. Acute idiopathic blind spot enlargement. A big blind spot syndrome without optic disc edema. Arch Ophthalmol 1988; 106: 44-9.

9 Horiguchi M, Miyake Y, Yagasaki K, Ichikawa K. Temporal aspects of the electroretinogram in cone-rod dystrophy. Doc Ophthalmol 1985; 60: 219-27.

10 Miyake Y. Studies of local macular ERG. Acta Soc Ophthalmol Fpn 1988; 92: 1419-49.

11 Miyake Y, Shiroyama N, Ota I, Horiguchi M. Oscillatory potentials in electroretinogram of the human macular region. Invest Ophthalmol Vis Sci 1988; 29: 1613-35.

12 Miyake Y, Shiroyama N, Horiguchi M, Ota I. Asymmetry of focal ERG in human macular region. Invest Ophthalmol Vis Sci 1989; 30: 1743-9.

13 Sigh K, Frank MP, Shults WT, Watzke RC. Acute idiopathic blind spot enlargement. A spectrum of disease. Ophthalmology 1991; 98: 497-502.

14 Miyake Y, Shiroyama N, Ota I, Horiguchi M. Local macular electroretinographic responses in idiopathic central serous chorioretinopathy. Am $\mathcal{f}$ Ophthalmol 1988; 106: 546-550.

\title{
Contralateral active ocular toxoplasmosis in Fuchs' heterochromic cyclitis
}

\author{
E La Hey, G S Baarsma
}

Department of

Ophthalmo-Immunology,

The Netherlands

Ophthalmic Research

Institute, Amsterdam

E La Hey

The Eye Hospital, Rotterdam, The

Netherlands

G S Baarsma

Correspondence to:

Dr Ellen La Hey, Departmen

of Ophthalmo-Immunology,

The Netherlands Ophthalmic

Research Institute,

Amsterdam, The

Netherlands.

Accepted for publication

19 February 1993
Many authors have published papers on the assumed association between Fuchs' heterochromic cyclitis and ocular toxoplasmosis. ${ }^{1-8}$ Most studies reported on the presence of chorioretinal scars which were clinically consistent with a previous intraocular toxoplasmosis. In the majority of cases these toxoplasmosis-like lesions were present in the cyclitic eye. Only a few patients with Fuchs' heterochromic cyclitis and active Toxoplasma retinochoroiditis have been described..$^{1-378}$ Until now, this clinical picture of toxoplasmosis was confirmed by aqueous humour analysis in two cases only. ${ }^{2}$ In a recent study we found a significantly higher incidence of toxoplasmosis-like chorioretinal scars in patients with Fuchs' heterochromic cyclitis than in a control group of patients with an HLA-B27positive anterior uveitis. ${ }^{6}$ Although we found this positive clinical association between Fuchs' heterochromic cyclitis and toxoplasmosis-like scars, it could not be substantiated by serological tests for toxoplasmosis (immunofluorescence or ELISA) or by a test for cellular immunity to Toxoplasma antigen. Analysis of aqueous humour samples for Toxoplasma antibodies also yielded negative results. One must keep in mind, however, that no active chorioretinal lesions were present in the patients with Fuchs' heterochromic cyclitis at the time of blood sampling, nor at the time when the aqueous humour samples were obtained (during cataract surgery) ${ }^{6}$

Here, we report on a patient with unilateral Fuchs' heterochromic cyclitis who developed an active toxoplasmosis of the contralateral eye, which could be proved by aqueous humour analysis.

\section{Case report}

A 28-year-old patient consulted our ophthalmology department with complaints of a diminished visual acuity of the left eye (6/6 on the right, hand movements (HM) on the left). Ophthalmic examination disclosed small white keratic precipitates scattered on the endothelium, $1+$ flare in the aqueous, diffuse iris stromal atrophy, no posterior synechiae, and evident heterochromia. A dense subcapsular cataract was present. Tests to exclude other causes of uveitis were all within normal range. Based on the clinical picture our diagnosis was Fuchs' heterochromic cyclitis. The right eye showed no anterior segment abnormalities, but fundus examination disclosed small pigmented chorioretinal scars nasally. During the cataract extraction we obtained aqueous humour of the left eye after informed consent. No Toxoplasma antibodies could be detected, even in undiluted aqueous humour. The Toxoplasma antibody titre in a paired serum sample was 1:32. No fundus lesions were seen after removal of the cataract. Two years later, this patient returned with a diminished visual acuity of the right eye $(6 / 12$ on the right, 20/24 on the left). The left eye still showed the typical clinical features of Fuchs' heterochromic cyclitis, as described above. The right eye showed mutton fat keratic precipitates, 